\title{
CHROMOSPHERIC NLTE-RADIATIVE-TRANSFER MODELING OF dMe STARS
}

P.M.Panagi(1), E.Hondeline(2), C.J.Butler(1), M.Rodonò(3).

(1) Arnagh (observatory, Armagh, Northern Ireland BT61 9DG.

(2) Liboratoire de Physique Stellatre et Planetaire, BP n10, 91371 Verrieres le Buisson, Cedex, France.

(3) Institulo di Astronomia, Universita degli St, !: I. 95125 Catania, Italy.

\begin{abstract}
:
The formation of the Lyman and Balmer lines of Hydrogen at densitics typical in dMe stars is shown to be very dependent on the atonic nodel used viz. the number of levels and line transitions. The effect of increasing the number of levels and line transitions on the line fluxes and ionization fraction is also discussed.
\end{abstract}

We have computed theoretical line profiles for the Lyman and Balmer series of Ilydrogen for a variety of model chromospheres and model atoms. The dMe chromospheres are essentially an extension of those used by Ciampapa et.al. (1982) and extculled to higher temperatures regimes. An electron temperature and clensity structure typical for late-type stars has been added at the base of the chromosphere, to minic the photosphere, following Mould (1976). The computer colle used was that of Carlsson described in Scharmer and Carlsson (1985), and modificd by him to include the technique of collisional-radiative switching (Hummer and Voels 1988) to enhance the convergence properties; a severe problem when including the Ly $\alpha$ transition in detail. Symmetric Voigt profiles have been calculated uncler the assumption of complete redistribution. Stark lroadening las been included using the approximation of Sutton (1978). All model computations assume a plane-parallel geometry and solar abundances.

For a given atmospleric temperature and density structure we contputed mainly emission line profiles and fluxes for a variety of model Ilydrogen atoms. Models with and without $\mathrm{Ly} \alpha$ were used to observe the effects of the Ly $\alpha$ radiation field on the line fluxes.

The number of levels and line transitions was then varied up to a maximum of 16 levels and 60 lines. 
IVe present graphically the results from computations of one such model chromosplece in the figures.

\section{RESULTS:}

1. Eflects of inclusion and omission of Lya.

The inclusion or omission of Ly $\alpha$ in detail does not affect the Balmer line fluxes significantly, ty pically alout $20 \%$. In the computation of purely Balmer line profiles the assumption of Ly $\alpha$ in detail balance is therefore a rassonalile approximation.

The liue: Huxes of the other computed Lyman series are however significintly chinged. 'They decrease typically by a factor of five.

'The neutral Hydrogen fraction, dominated by the ground state population, decreases at tomperatures above $8000 \mathrm{~K}$, when Ly $\alpha$ is assumed to be in detiiled balince. When Lyo is excluded, radiative decay from level 2 is inhibited, so that the gromul state population is relatively depopulated. As a conseguence the higher levels are overpopulated giving rise to stronger emission lines in the ligher series members. The fraction of II II is relatively depleted at temporatures below $8000 \mathrm{~K}$, as are all the upper levels of II I. This will affect the ionization balance and subsequently the formation of other atomic: lines, as IIydrogen is the main donor of electrons in the chromosphere.

2. Effects of including more levels.

The inchusion of more levels in the atomic model also has a marked effect on the line fluxes, particularly on the Balmer series fluxes which decreased by a factor of two for Ho to nearly a factor of ten for $\mathrm{H}_{\gamma}$ in all our models.

Increasing the number of levels gives rise to a relative depopulation of the levels from which these lines are formed, as there are then more excitation paths. The II II population is also relatively depleted, markedly at the lower temperatures. Again this will have a strong effect or the ionization equilibrium and formation of lower chromospleric lines.

We finally note that the frequency quadratures at which the the line profiles are calculated has also been found to be of importance. The inclusion of more extended wings tends to bring the line peak to a higher value, typically $10 \%$, so that some wing photons are eventually trapped in the core. 


\section{References}

Giampapa M.S., Worden S.P., Linsky J.L.: Ap.J., 258, 7.10 (1982)

Hummer D.G., Voels S.A.: A.A. 192, 27 ? (1988)

Mould J.R.: A.A., 48, 443 (1976)

Scharmer G.B., Carlsson M.: J.Comp.Phys., 59, 56 (1985)

Sutton K.: J.Q.S.R.T. 20), 333 (1978)
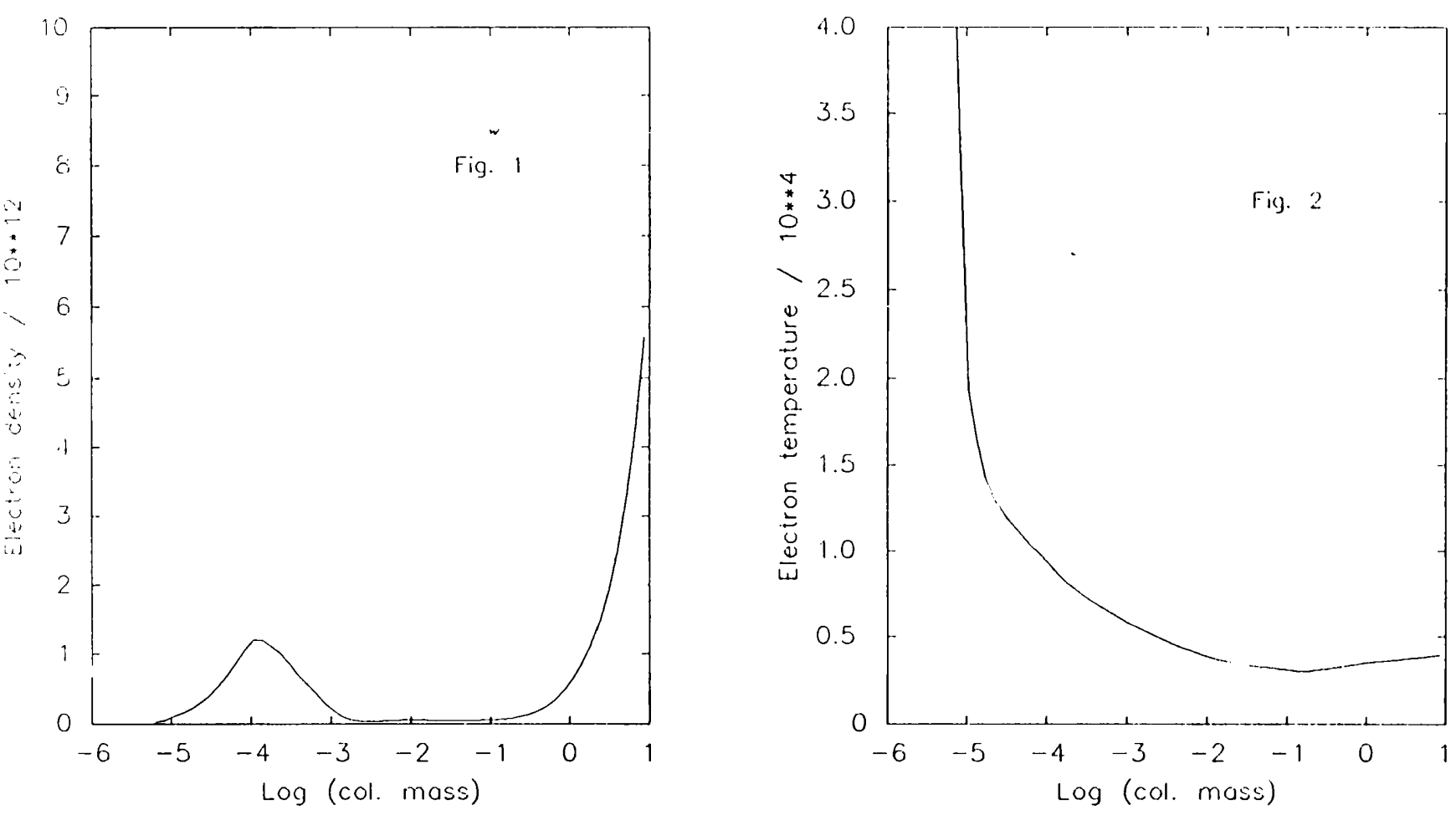

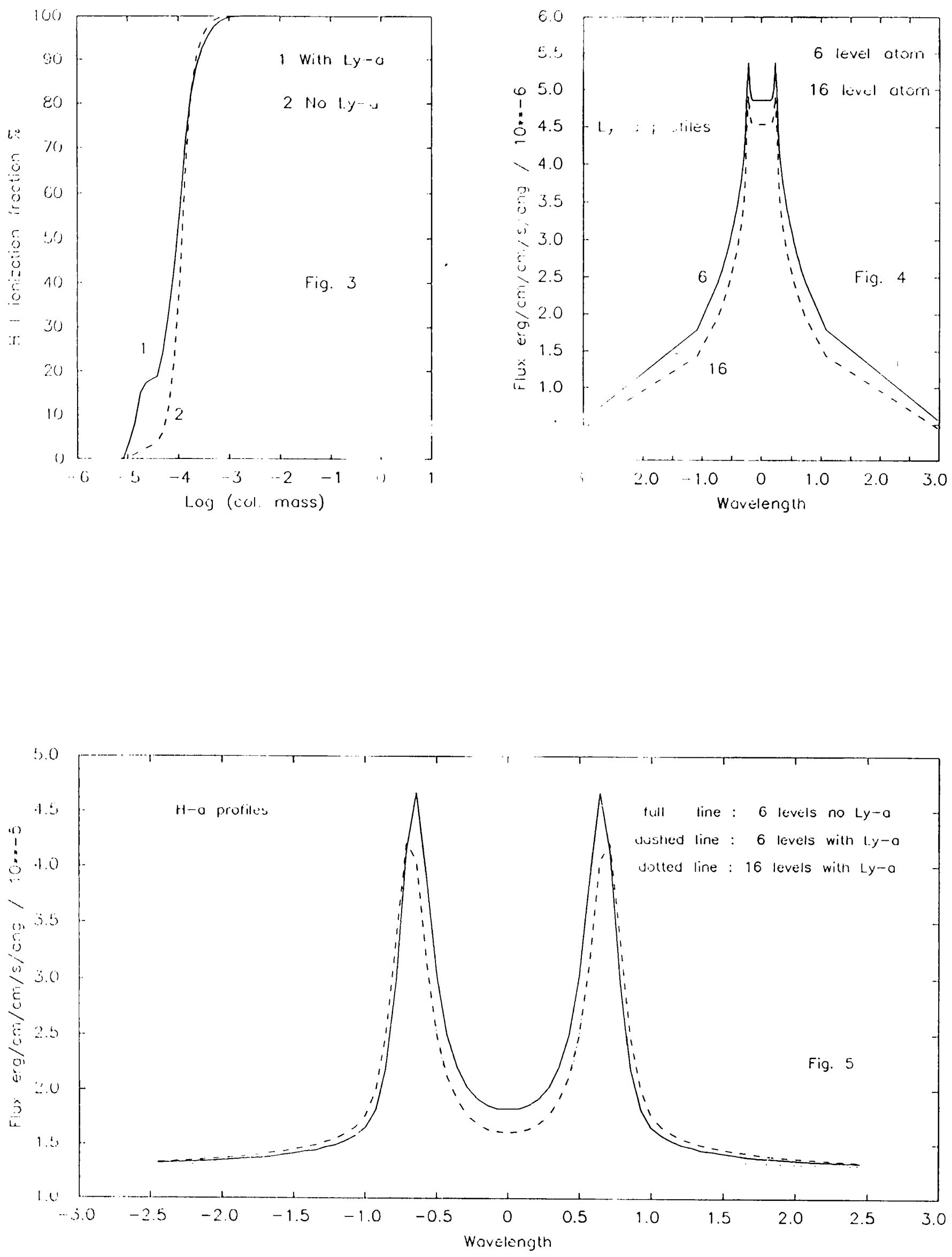